Aus der Inneren Abteilung der Krankenanstalt Altstadt in Magdeburg. (Oberarzt: Dr. E. Schreiber.)

\section{Ueber die Fehlerquellen der Methode der Opsoninbestimmung nach Wright.}

\author{
Von Dr. W. Beyer, Sekundärarzt.
}

Im Vergleich zu England und Amerika hat die Wrightsche Methode der Opsoninuntersuchung bisher in Deutschland noch geringen Eingang gefunden. Und während Wright sie von Anfang an für die Praxis eingerichtet hatte, beschäftigte sich die Mehrzahl der deutschen Autoren vorwiegend mit der theoretischen Seite der Frage und mit der Stellung, welche die Opsonine im System der Immunkörper einnehmen. So interessant diese Fragen auch sein mögen, ungleich wichtiger ist es jedoch zweifellos, sich zu fragen: Was kommt für die Praxis, zum wenigsten für die klinische, bei dieser Methode heraus? Bei Durchsicht der bisher erschienenen Arbeiten vermißt man leider oft genug das für die Beurteilung einer neuen Methode vorauszusetzende Maß der Kritik. Die Mehrzahl der Arbeiten sind zudem nur allgemeine Referate, manche davon enthalten ausgedehnte Beschreibungen der Technik und sind zum Teil mit einem aufallenden Enthusiasmus geschrieben, der zuweilen an Stelle eigener objektiver Ergebnisse tritt. Wieder andere Arbeiten gehen ohne weiteres frisch daran, die Methode auf die Diagnose und Therapie aller möglichen Krankheiten anzuwenden, ohne sich vorher über ihre Fehlerquellen genügend klar geworden zu sein. Nimmt man dazu, daß viele Untersucher die Technik nur aus der Literatur gelernt haben und teilweise, wie aus manchen Bemerkungen hervorgeht, nicht sehr vollkommen beherrschen - während auf der andern Seite diese Technik eingestandenermaßen so schwierig ist, daß auf einem amerikanischen Kongreß ein Autor allen Ernstes behauptet hat, ohne eine mindestens fünfjährige Uebung könne einer in dieser Frage garnicht mitreden, so muß man zugeben, daß es vor allen Dingen darauf ankommt, und zwar für jeden einzelnen Untersucher, erstens festzustellen, wie weit sich die Methode als solche in seiner Hand als zuverlässig erweist; und zweitens, wenn dies nicht der Fall, liegt der Grund beim Untersucher oder außerhalb desselben? Ist eine hinreichend Verläßlichkeit der Methode sichergestellt, so ergeben sich dann weiter die beiden Fragen nach der Konstanz der "Normal“Opsonine des gesunden Menschen und nach der Spezifizität der „Immun"-Opsonine bei Infektionen. Erst wenn diesen Postulaten genügt ist, kann jemand diese Methode mit Recht auch anderen empfehlen.

Ich sehe hier von einer Schilderung der Wrightschen Technik, ${ }^{1}$ ) welche ich im Frühjahr des Jahres in seinem Laboratorium studiert habe, ab. Sie ist zwar sehr originell und elegant, jedoch ohne persönliche Unterweisung meiner Ansicht nach kaum genügend zu erlernen. Anderseits ist ein leichtes und sicheres Beherrschen des Technischen hier die unerläßliche Vorbedingung für einwandfreie Resultate, und man muß anfangs reichlich Geduld haben, bis sich allmählich die nötige Geschicklichkeit einstellt. Liegt nun der gut gelungene und sauber gefärbte „Film" unter dem Mikroskop, so sei nun, sollte man meinen, nichts leichter, als eine beliebige Anzahl von Leukozyten, welche alle an einem Rande liegen, samt den von ihnen "gefressenen" Mikroorganismen durchzuzählen. Dem ist jedoch nicht so. Sind die Kokken oder Bakterien gut gefärbt, was bei alten Laboratoriumsstämmen oft nicht möglich ist, ohne gleichzeitig die Leukozyten zu überfärben und dadurch die Zählung erheblich zu erschweren, so ist es, wie unter andern Jürgens hervorhebt, oft recht schwer zu entscheiden: was liegt innerhalb der Zellen und ist mitzuzählen und was nicht. Nur öftere Betrachtung verschieden stark gefärbter Präparate sowie Innehaltung stets der gleichen Gepflogenheiten beim Zählen helfen über diese Schwierigkeiten hinweg. Als ich noch bei Wright arbeitete, fiel es mir auf, daß ich beim Durchzählen von Präparaten seiner Assistenten öfter Zahlen

1) Vergleiche hierzu den ausführlichen Aufsatz voa Strubel in No. 19 dieser Wochenschrift 1908. D. Red. erhielt, welche von den ihrigen ziemlich erheblich differierten, und zwar nicht nur absolut, sondern auch relativ, beim Ausrechnen der opsonischen Indices. Ich schob dies anfangs auf mangelnde Uebung meinerseits, mußte mich jedoch später überzeugen, daß der Grund noch anderswo lag.

Avfangs begnügte sich $\mathrm{W}$ right bekanntlich damit, je 30 Leukozyten auszuzählen; später stieg er jedoch mit der Zahl, und zurzeit werden in seinem Institut meist 50, zuweilen 100 Leukozyten ausgezählt. Wright und seine Schule geben an, daß die in der Natur der Sache liegenden Fehlergrenzen für geübte Untersucher 5 bis allerhöchstens $10 \%$ betragen. $10 \%$ angenommen, dürfte also z. B. eine erstmalige Auszählung von 50 Leukozyten in einem Präparat 450, eine nochmalige allerhöchstens 500 intrazelluläre Bazillen ergeben. Man kann eine entsprechende Kontrolle auch einfach so vornehmen, daß3 man hintereinander im Präparat weiterzählt, und zwar ohne Ausnahme alle vielkernigen Zellen, die bei langsamem, stetigem Weiterschieben des Präparates das Gesichtsfeld passieren. So kann man bequem 100 Zellen und mehr auszählen, ohne sich vom Ausgangsbezirk erheblich zu entfernen. Den von Wright angegebenen $10 \%$ gegenüber betont eine Reihe amerikanischer Autoren, daß die Fehlerquellen in Wirklichkeit erheblich größer seien. So betrug nach Potter die durchschnittliche Abweichung in 92 von demselben Untersucher doppelt gezählten Staphylokokkenpräparaten $9,8 \%$, die maximale dagegen bis $z u 34,1 \%$ in ein und demselben Präparat. 57 andere Präparate lieferten in der Hand eines anderen Zählers durchschnittlich $12,3 \%$, im Maximum $36 \%$, 24 Tuberkelbazillenpräparate sogar bis zu $110 \%$ Differenzen. Zwölf andere Tuberkelbazillenpräparate wurden von zwei Untersuchern nacheinander gezählt: die durchschnittliche Differenz betrug $24 \%$, die maximale $51 \%$. Ebenso ergaben sich Differenzen in den verschiedenen Bezirken eines und desselben Präparates. $\mathrm{Zu}$ ähnlichen Resultaten gelangten Moss, Park und Biggs, Bolduan, Thomas. Von deutschen Autoren sah Weinstein beträchtliche Differenzen, die er subjektiven Momenten zur Last legt. Einer eingehenden Kritik unterzog Sa a th off die Technik. Er fand Differenzen der Zahlen bis zu 50\% zweier Proben desselben Serums, wenn er je 50, und bis zu $33 \%$, wenn er je 100 Leukozyten zählte. Meine Erfahrungen sind folgende:

In Präparaten mit Typhus und Paratyphus-Emulsionen von ziemlicher Dichtigkeit und Serum verschiedener nicht spezifisch kranker Menschen zählte ich je zweimal 50 Leukozyten, wie oben geschildert, hintereinander. Die Differenzen der beiden erhaltenen Zahlen betrugen $4,10,10,13,43 \%$; in einer andern Serie 2, 4, 22, 22, $26 \%$; in einer dritten $19,26,28,33,42,44 \%$. Wenn beispielsweise die ersten 50 Leukozyten 1384, die zweiten 1078 Bazillen einschlossen, so ergibt das eine Differenz von $28 \%$. Die durchschnittliche Differenz betrug also $22 \%$ die größte $44 \%$ die kleinste $2 \%$. Nun sind gerade Laboratoriumsstämme von Typhus nicht ideal für solche Versuche, insofern Bakteriolyse und Agglutination, welch letztere in diesen Präparaten indes nur sehr schwach auftrat, die Beurteilung immerhin etwas erschweren. Versuche mit Diplococcus lanceolatus ergaben analog ausgeführt folgende Differenzen: $3,10,16$, $22,26,27,29,30,41 \%$. Um die Dichtigkeit der hierzu verwendeten Emulsion zu schätzen, sei bemerkt, daß der Durchschnitt von gefressenen Kokkenpaaren $1-5$ pro Leukozyt betrug, während die Kapillaren zwölf Minuten wagerecht im "Opsonizer" verweilten. Weitere Proben machte ich mit einem Stamm von Staphylococcus albus, der aus dem Blute eines Sepsisfalles frisch gezüchtet und in der zweiten Agargeneration benutzt wurde. Alter der Kultur 48 Stunden, Kapillaren 15 Minuten im Brutapparat. Fixierung der trockenen Ausstriche mit gesättigter Sublimatlösung, Färbung mit $0,5 \%$ Methylenblau-Sodalösung 4 Minuten. Durch mehrmaliges Abspülen und dazwischen eingeschobene Pause wurde eine außerordentlich distinkte Färbung und in jeder Hinsicht tadellose Präparate erzielt. Die Zahl der von je j0 Leukozyten gefressenen Kokken betrug im Präparat:

\begin{tabular}{|c|c|c|c|c|}
\hline \multirow[b]{2}{*}{ Präparat } & \multicolumn{4}{|c|}{ Anzahl der Kokken } \\
\hline & 1. & 395 & 476 & 415 \\
\hline & ll. & 304 & 267 & $26 t$ \\
\hline & lll. & 206 & 149 & \\
\hline & IV. & 415 & 354 & \\
\hline & V. & 384 & 350 & \\
\hline & Vl. & 501 & 428 & \\
\hline & Vil. & 248 & 228 & \\
\hline & Vill. & 302 & 262 & \\
\hline & IX. & 404 & 277 & \\
\hline & $\mathrm{X}$. & 404 & 270 & \\
\hline & $\mathrm{Xl}$. & 462 & 414 & \\
\hline & Xll. & 365 & 322 & \\
\hline
\end{tabular}

$$
\begin{aligned}
& \text { Differenzen im selben Präparat } \\
& =20 \% \quad 15 \% \\
& =14 " 5 \% \\
& =38 " 1 \% 14 \\
& =17 n \\
& =9 " \\
& =17 n \\
& =9 n \\
& =15 n \\
& =46 " \\
& =50 " \\
& =11 " \\
& =13 \%
\end{aligned}
$$

Hier sind gleichfalls Differenzen bis zu $50 \%$ in einem und demselben Präparat vorhanden. $\mathrm{Zu}$ einer weiteren Versuchsreibe verwendete ich Anthraxbazillen: 


\begin{tabular}{|c|c|c|c|}
\hline \multirow{10}{*}{ Präparat } & I. & 311 & $304=2 \%$ Differenzen \\
\hline & II. & 263 & $243=8 n$ \\
\hline & III. & 362 & $288=26 n$ \\
\hline & IV. & 213 & $196=9 n$ \\
\hline & v. & 225 & $195=15 n$ \\
\hline & VI. & 173 & $106=63 n$ \\
\hline & VII. & 474 & $321=48 n$ \\
\hline & VIII. & 324 & $180=80^{?}$ \\
\hline & IX. & 283 & $276=3 \%$ \\
\hline & $\mathrm{X}$ & 555 & $463=20$ " \\
\hline
\end{tabular}

Also auch hier wieder erhebliche Differenzen. Wright hat früher empfohlen, Leukozyten mit beträchtlich über den Durchschnitt herausragender Mikrobenzahl von der Zählung auszuschließen. Später hat er jedoch diese Forderung derart eingeschränkt, daß er Z. Z. bei einem Durchschnitt von 4-5 Mikroben als Höchstzahl 30 gelten läßt, bei Tuberkelbazillen nur 9-10. Wenn auch vielleicht der erstere Spielraum etwas hoch erscheint, so ist er doch gerechtfertigt, wenn die Emulsionen kunstgerecht hergestellt sind und die Mikroben nicht klumpenweise, sondern einzeln im Leukozyten liegen. Ich folgte im übrigen durchaus den Methoden, die ich am Wrightschen Institut erlernt hatte und in deren gleichmäßiger Uebung ich die nötige Sicherheit seit längerer Zeit besaß. Nach meinen Untersuchungen also betragen die Fehlergrenzen der Methode bis zu $80 \%$, wenn man nur 50 Leukozyten auszählt. Danach würden also Schwankungen im opsonischen Index z. B. zwischen 0,55 und 1,80 noch ins Bereich der unvermeidbaren Fehlerquellen fallen, wenn man von 2 um $80 \%$ differierenden Zahlen die eine in die andere dividiert, wie das bei Ausrechnung des opsonischen Index geschieht. Nimmt man je 100 Leukozyten, so werden die Fehlerquellen etwas geringer und bei 200 noch etwas geringer. Immerhin bleiben sie auch so noch erheblich genug, wie folgende Zahlen zeigen. In einem Präparat zählte ich 500 Leukozyten aus. Die Zahl der Staphylokokken in je 50 Zellen betrug: $268,289,372,242,256,277$, 235, 228, 355, 291. Die maximale Differenz zwischen je zwei dieser Zahlen beträgt $63 \%$. Kombiniert man von diesen zehn Zahlen je zwei, eine Summe, welche also je 100 Leukozyten entspricht, so bekommt man eine Maximaldifferenz von $57 \%$, dagegen von $39 \%$, wenn man nur die einander benachbarten Zahlen (benachbarten Bezirke im Präparat) zusammenaddiert. Kombiniert man ron diesen fünf Summen je zwei zu einer neuen Zahl, die also je 200 Leukozyten entsprechen würde, so bliebe immer noch eine Maximaldifferenz von $37 \%$.

Aus den eben mitgeteilten Resultaten geht hervor, daß die Fehlerquellen der Technik der Opsoninbestimmung derart erhebliche sind, dal nur Werte, die sich um mehr als fast das Doppelte voneinander unterscheiden, einen Schluß auf Differenzen im Opsoningehalt des Serums zulassen können, kleinere Unterschiede jedoch von vornherein solche Schlüsse verbieten. Sind diese Ergebnisse richtig, so schränken sie die Verwertbarkeit der Methode bedeutend ein. Es fragt sich nun, wie es mit der angenommenen Konstanz der "Normal"opsonine steht, welche eine weitere Voraussetzung der Opsonintheorie bildet, indem ja das Immunserum gegen ein einzelnes oder gemischtes Standardserum von Gesunden ausgewertet wird. Finden sich hier Differenzen der einzelnen Sera noch unter $80 \%$, so beweisen sie nach dem oben Mitgeteilten natürlich noch nichts gegen eine Konstanz, sind sie erheblich größer, so beweisen sie allerdings etwas dagegen, vorausgesetzt daß sich die Fehlerquellen beim Vergleich von Präparaten aus verschiedenen Kapillarröhren nicht noch wesentlich steigern. Dies ist nicht der Fall, wie ich durch Versuche mit demselben Serum, aber verschiedenen Kapillarröhren feststellte. Die oben verzeichneten Versuche machte ich zugleich, um der Frage der Konstanz der Opsonine bei Gesunden näher zu treten.

Ich entnahm gesunden Personen mittels Wrightscher Glasspindel von der Dorsalseite des Fingers zur selben Zeit Blut und untersuchte es gleichzeitig hintereinander mit derselben Mikrobenemulsion. Die Kapillarröhren, deren Kaliber so gleichmäßig wie möglich ausgesucht wurde, blieben bei Staphylokokken immer genau 15, bei Diplokokken 12, bei Anthrax 8 Minuten im Inkubator, die weitere Behandlung, Fixierung und Färbung geschah ebenfalls gleichmäßig. Nimmt man aus den oben angeführten Versuchen mit Staphylokokken die mittleren Werte, also je 100 Leukozyten entsprechend, so ergeben sich, nach der Größe geordnet, folgende Ziffern: $355,476,564,571,674,681,687,734,769,871,876,929 . \quad$ Die "phagozy tische Zahl“ variiert also hier zwischen 3,55 und 9,29, d. h. bis um $162 \%$. Bei Benutzung der Zahlen für je 50 Leukozyten sind es sogar $236 \%$. Noch verschiedener waren dieWerte für Milzbrandbazillen, zu welchen ich Serum von den gleichen Personen an einem andern Tage wiederum gleichzeitig entnahm und untersuchte. Die phagozytische Zahl differierte von 2,8 bis zu 10,1, also um mehr als das Dreifache, oder um $260 \%$. Die entsprechenden Zahlen für je 100
Leukozyten waren: 279, 409, 420, 504, 506, 559, 615, 650, 795, 1018. Versuche mit Diplococcus lanceolatus ergaben folgende Zahlen: 166, $194,221,242,356,381,442,461,463$.

Also auch hier Schwankungen der "Normal"opsonine um fast das Dreifache. Würde man nun z. B. eine StandardMischung aus drei verschiedenen Seris herstellen, so ist klar, daß hierdurch keineswegs eine Garantie gegen abnorme Werte geboten wird. Ganz abgesehen davon, ob man überhaupt erwarten darf, durch solche Mischungen wirklich auch die Mittelwerte der Komponenten zu erhalten (vgl. Simon, Lamar, Bispham) - man braucht nur an einen verschiedenen Gehalt von Ambozeptoren und Komplementen zu denken -, so wäre es reiner Zufall, Sera herauszugreifen, die sich kompensieren und nicht nach derselben Richtung exzedieren. Schlieflich entnahm ich noch gesunden Personen Serum zu verschiedenen Tageszeiten und untersuchte die Phagozytose auf Staphylokokken.

Für die folgende Tabelle wurden von jeder Probe, d. h. Mischung in einer Kapillarröhre, immer zwei Ausstriche hergestellt, von welchen im Minimum je 200, in mehreren Präparaten je 400 Leukozyten ausgezählt wurden.

\begin{tabular}{|c|c|c|c|}
\hline \multirow[b]{2}{*}{$\begin{array}{l}\text { Zeit der Serum- } \\
\text { Entnahme }\end{array}$} & \multicolumn{3}{|c|}{ Zahl der in je 200 Leukozyten eingeschlossenen Kokke } \\
\hline & I. Ausstrich. & II. Ausstrich & $\begin{array}{l}\text { Summa }=\text { auf je } \\
400 \text { Leukozyten }\end{array}$ \\
\hline 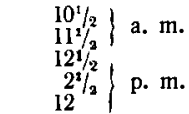 & $\begin{array}{l}404 \\
834 \\
754 \\
471 \\
624\end{array}$ & $\begin{array}{l}393 \\
900 \\
773 \\
582 \\
615\end{array}$ & $\begin{array}{l}797 \\
1734 \\
1527 \\
1053 \\
1239\end{array}$ \\
\hline
\end{tabular}

Ein Blick auf diese Tabelle zeigt, daß hier unregelmäßige Schwankungen vorkommen, da die auf je 400 Leukozyten berechneten Zahlen unter sich bis um mehr als das Doppelte differieren.

Schluß. Vergleicht man mit den hier dargelegten, in der Methode - im engeren und weiteren Sinn - begründeten großen Differenzen die oft um ein Vielfaches kleineren Ausschläge der Resultate und opsonischen Kurven bei Infektionskrankheiten, so hat man in der Tat den Eindruck, daß derjenige, der aus jenen kleinen Differenzen weitgehende Schlüsse auf den opsonischen Gehalt des Serums ziehen wollte, ähnlich wie jemand verfahren würde, der es unternimmt, auf einer ungleich mit Balken belasteten Wage Splitter abzuwiegen. Um somit zu schließen, so haften der Methode derartige Fehlerquellen an, daß sie, ganz abgesehen von dem unverhältnismäßig großen Aufwand an Zeit, Geduld und Mühe, für klinische Zwecke, wenigstens in ihrer jetzigen Form, so gut wie unbrauchbar sein dürfte.

Literatur: Bolduan, ref. Bull. Part. 1908, No. 3. - FIeming, Practitioner, May 1908. - Jü rgen s, Berliner klinische Wochenschrift 1908, No. 13. Moss, Studies of Opsonins, John Hopk. Hosp. Bull. 1907, No. 195. - Ne u f el d Opsonine und Bakteriotropine in Kolle-Wassermanns Handbuch 1908. - Park and B i g g s, Journ. med. reseach. Oct. 1907, S. 77. - Pot te r, Journ. of Amer. med. Ass 30. Nov. 1907, S. 1815. - S a a t h of $\mathrm{f}$, Münchener medizinische Wochenschr. 14. April 1908. - Sim o n, L a m a r, B i s ph a m, Journ. of exp. med., Bd. 8, S. 65I. - Th o. $\mathrm{m}$ a s, Journ. of Amer. med. Ass. 12. Oct. 1907, S. 1249. 